\title{
High Rates of Anxiety Among Adolescents in a Partial Hospitalization Program
}

\author{
Michelle Pelcovitz $^{1}$ (D) . Shannon Bennett ${ }^{1} \cdot$ Payal Desai $^{2} \cdot$ Jennifer Schild ${ }^{1}$. \\ Renae Beaumont ${ }^{1} \cdot$ John Walkup ${ }^{3} \cdot$ David Shaffer $^{4} \cdot$ Angela Chiu $^{1}$
}

Accepted: 3 February 2022 / Published online: 24 February 2022

(c) The Author(s), under exclusive licence to Springer Science+Business Media, LLC, part of Springer Nature 2022

\begin{abstract}
Background Anxiety disorders are garnering increasing attention for their contribution to high-risk issues and functional impairment. Adolescents are typically admitted to partial hospitalization programs (PHPs) due to high-risk presentations. However, the frequency of anxiety disorders in PHPs is not well-established, in part because anxiety can be overlooked in acute settings due to limited lengths of stay and focus on stabilization.

Objective This study aims to evaluate the frequency and severity of anxiety disorders among a sample of adolescent PHP patients to assess the need for anxiety-specific assessment and interventions in higher acuity settings.

Methods Participants were 158 youths ages 13 to 19 years old $(M=15.49$ years, $S D=1.50)$ who were admitted to an adolescent PHP and their caregivers. Clinician-reported diagnostic information was collected from the youth's electronic medical record, and self- and caregiver-rated severity of anxiety was collected using the Screen for Child Anxiety Related Emotions Disorders (SCARED-C/P). Frequency of anxiety and related disorder diagnoses and self- and caregiver-reported severity were assessed using descriptive statistical methods. Results $75 \%$ of participants were diagnosed with an anxiety disorder $(n=118)$. On average, participants with anxiety disorders had elevated SCARED-C scores. Youths with depressive disorders had elevated SCARED-C scores even when they did not carry anxiety disorder diagnoses. Caregiver ratings of the youth's anxiety symptoms on the SCARED-P were elevated when youths had anxiety disorders.

Conclusions These findings suggest that anxiety is common in an adolescent PHP setting and support investing in evidence-based assessment and treatment of anxiety in high-acuity settings.
\end{abstract}

Keywords Anxiety $\cdot$ Adolescence $\cdot$ Symptom severity $\cdot$ Acute care

Anxiety disorders are the most common class of psychiatric diagnosis in the U.S (Kessler et al., 2012) and adolescence confers the highest risk for developing an anxiety disorder (Merikangas et al., 2010). In the context of the COVID-19 pandemic, these numbers appear

Extended author information available on the last page of the article 
to be rising precipitously (Courtney et al., 2020; O'Sullivan et al., 2021). Anxiety disorders are often associated with concerning and sometimes severe functional impairments (Craske et al., 2017; Woodward \& Fergusson, 2001), which can lead to the need for a higher level of care. Unrecognized, untreated, or under-treated anxiety disorders can contribute to severe functional impairment and disability (Baxter et al., 2014). However, despite the high prevalence rates and risk of disability from anxiety disorders in this age range, anxiety often gets inadequate attention in higher levels of care.

In the literature, anxiety disorders are sometimes misrepresented as mild, only treated in outpatient settings and are perceived as rare or insignificant in inpatient settings (e.g., Bandelow \& Michaelis, 2015). On the contrary, anxiety is the sixth leading cause of disability worldwide (Baxter et al., 2014) and thus undoubtedly contributes to the need for higher levels of care. Understanding the presentations of anxiety among youths who present to intensive psychiatric services may help to clarify the need for higher levels of care to expand or intensify evidence-based anxiety interventions to decrease suffering, improve overall outcomes, and decrease risk of rehospitalization.

Partial hospitalization programs (PHPs) are an intermediary treatment option within the psychiatric continuum of care for patients who require more clinical attention than weekly outpatient care can provide but offer less intensive services and a less restrictive setting than inpatient hospitalization (Mee-Lee \& Shulman, 2003). Substance Abuse and Mental Health Services Administration (2019) analyzed data from the 2018 National Mental Health Services Survey (N-MHSS) and found that $23.2 \%$ of the 902,665 children and adolescents seeking outpatient psychiatric care in 2018 were served in PHP or day treatment programs.

Unfortunately, research detailing the use of evidence-based interventions in PHP settings is scant. Treatment approaches appear to vary widely between programs, and most often focus on the treatment of eating disorders, disruptive behavioral disorders, or mood disorders (e.g. Leffler et al., 2020; Robinson, 2000). Robinson and colleagues (1999) describe adopting a predominantly behavioral approach to their partial program, with opportunities to generalize skills throughout the day within the milieu. Dialectical behavior therapy (DBT) and cognitive behavior therapy (CBT) skills have also been used in group-based programming in PHP settings (e.g. Del Conte et al., 2016; Martino et al., 2020), however the ability to best apply interventions to specific anxiety, mood, or related symptoms first requires careful assessment and treatment planning of all primary and comorbid conditions. Better understanding the assessment, treatment, and common presentations of patients in PHP settings is essential to optimize outcomes and improve personalized care. Moreover, data reflecting rates of common diagnoses in a PHP setting is of value to outpatient, emergency, and inpatient clinicians who make and receive referrals to and from this level of care.

The reasons for admission to PHPs are typically high-risk issues, such as suicidal ideation, suicide attempts, non-suicidal self-injury, or severe behavioral problems. As such, these settings must prioritize those presenting problems, often to the exclusion of other psychiatric diagnoses and presentations. At present, the frequency of anxiety disorders in partial hospitalization and acute care settings is not well established. A meta-analysis examining global prevalence of youth psychiatric disorders found that of the 241 million youths around the world that are affected by a mental disorder, 117 million youths are diagnosed with anxiety disorders, making it the most common category of diagnosis, regardless of level of psychiatric care (Polanczyk et al., 2015). Although initial findings suggest that the frequency of anxiety disorders can be high in adolescent PHPs (Thatte et al., 2013), these 
results have relied on a small sample size $(n=55)$. Other studies that have included larger samples have reported much lower rates (e.g., 13\%) of anxiety disorders among youths treated within these settings (Robinson, 2000). Studies have all largely relied on admission diagnoses. Impressions at the intake may not be as sensitive to the identification of anxiety disorders relative to diagnostic information gathered from discharge summaries, which are more likely to reflect the team's impressions after more time has passed to observe, assess, and get to know the constellation of problems impacting the youth. Thus, it is worthwhile to assess the specific rates of anxiety and related disorders not only at admission but also upon discharge from adolescent PHPs.

Furthermore, the relationship between anxiety and depression in youth is multi-faceted and in need of further study to optimize assessment, treatment, and prevention. Depressive disorders are thought to be the common cause of risk behaviors leading to referral of youth to higher levels of care (e.g., Gould et al., 2003; Hallfors et al., 2004; Shaffer et al., 1996); however, it is apparent that the comorbidity of anxiety and depressive disorders is a nuanced phenomenon with multiple explanatory pathways (e.g., Cummings et al., 2014). For example, youths may feel anxious secondary to being depressed or may develop depression as a consequence of living with anxiety. Moreover, global deficits in affect regulation, distress tolerance and/or behavioral activation put youths at risk for both anxiety and depression (Trosper et al., 2009). As such, understanding this complex and nuanced relationship, which likely plays a role in PHP patients' presenting problems, should be explored.

There are documented limitations in comprehensive diagnostic assessment in high acuity psychiatric settings, particularly for anxiety and related disorders, in the absence of using a formal structured or semi-structured interview (Zander et al., 2018). Several factors might contribute to the scarcity of diagnostic data in acute care settings. On a fast-paced unit with short lengths of stay, assessment must be rapid, with a premium placed on prioritizing safety and stabilization. Clinicians may also have limited time, resources, or experience for structured diagnostic interviews. Continued omission of anxiety disorders from the treatment plan and/or follow-up care plan due to failure to accurately recognize, diagnose, and prioritize anxiety, may increase risk for relapse and possibly re-hospitalization given the significant morbidity associated with untreated or under-treated anxiety disorders (NaragonGainey et al., 2014). In the absence of gold standard diagnostic procedures, this study sought to learn more about the frequency and severity of anxiety disorders and symptomatology by relying on two sources of information: diagnostic impressions from the electronic medical record and self-report measures.

A clearer understanding of the frequency and severity of anxiety as a primary presenting problem among adolescent PHP patients would justify creating more robust programming to target anxiety in this setting. Additionally, understanding and identifying the various presentations of anxiety and depressive disorders is essential to optimize treatment planning. Therefore, the primary aim of this study was to assess the frequency of anxiety and related disorders, including comorbid depressive disorders, among a sample of youths presenting for PHP services using medical record data. The second aim of this study was to examine anxiety symptom severity in the PHP using self- and parent-report data. 


\section{Method}

\section{Participants}

Participants were 158 youths ages 13 to 19 years old $(M=15.49$ years, $S D=1.50)$ who were admitted to an adolescent PHP located in a metropolitan academic medical center in Northeastern U.S. between August 5th, 2016, and December 31, 2018. Caregivers of the youths also participated. Slightly more than half $(51.3 \%)$ of the participants were referred by regional psychiatric inpatient facilities. The remaining participants were referred by outpatient providers $(40.6 \%)$, emergency rooms $(8.1 \%)$, and residential or day treatment programs (less than 1\%). At the end of their PHP stay, the majority $(89.0 \%)$ of participants were discharged to outpatient providers in the community. Only a small proportion were discharged to the emergency room (1.8\%), recommended for inpatient admission $(4.30 \%)$, referred to a day treatment program $(3.7 \%)$ or to a residential program $(1.2 \%)$. Across selfreported sociodemographic data, $7.0-13.9 \%$ did not provide a response. Parent report of

Table 1 Sample Characteristics

Note: GED = General education

\begin{tabular}{|c|c|c|}
\hline Sociodemographics & $n$ & $M(S D) / \%$ \\
\hline Age & 158 & $15.49(1.50)$ \\
\hline \multicolumn{3}{|l|}{ Sex (assigned at birth) } \\
\hline Male & 51 & 35.9 \\
\hline Female & 91 & 64.1 \\
\hline \multicolumn{3}{|l|}{ Gender identity } \\
\hline Male & 50 & 35.0 \\
\hline Female & 86 & 60.1 \\
\hline Transgender & 2 & 1.4 \\
\hline Gender nonconforming & 2 & 1.4 \\
\hline Queer & 1 & 0.7 \\
\hline Questioning & 1 & 0.7 \\
\hline Other & 1 & 0.7 \\
\hline Declined to state & 0 & 0.0 \\
\hline \multicolumn{3}{|l|}{ Ethnicity } \\
\hline Hispanic & 33 & 24.3 \\
\hline Non-Hispanic & 103 & 75.7 \\
\hline \multicolumn{3}{|l|}{ Race } \\
\hline White/Caucasian & 86 & 60.6 \\
\hline African American & 12 & 8.5 \\
\hline American Indian & 2 & 1.4 \\
\hline Asian & 14 & 9.9 \\
\hline More than one race & 17 & 12.0 \\
\hline Other & 11 & 7.7 \\
\hline \multicolumn{3}{|c|}{ Parental highest education level } \\
\hline No high school & 1 & 0.7 \\
\hline High school or GED & 12 & 8.2 \\
\hline College or technical school & 57 & 38.8 \\
\hline Post graduate degree & 77 & 52.4 \\
\hline
\end{tabular}


race and ethnicity was used when youth report of race and/or ethnicity was missing. Of those in the sample that self-reported sociodemographic data, more than half were female (64.1\%) and non-Hispanic (75.7\%). More than half (60.6\%) of the sample was Caucasian, and $12.0 \%$ of participants self-identified as belonging to more than one race. Of the 135 caregivers who completed the intake questionnaires, $52.6 \%$ were mothers, $20.0 \%$ fathers, $14.1 \%$ both parents, $2.2 \%$ other family members (e.g., sister, grandmother), and $11.1 \%$ did not report their relation to the youth participant. Caregivers tended to be highly educated as $52.4 \%$ had post-graduate degrees and $38.8 \%$ had college degrees listed as their highest level of education. See Table 1 for additional demographic details about the sample.

\section{Procedures}

Study procedures were approved by the academic medical center Institutional Review Board and conducted in accordance with ethical standards. All youths who were admitted to the adolescent PHP with at least one English-speaking caregiver were invited to participate in this study, which was part of a larger clinical data repository initiative. Youths and their caregivers were approached for the study during their stay in the PHP. Unless the youth was 18 years old, youth participants were included in the study only when the caregiver signed consent and the youth provided assent to participate.

All youths and their caregivers completed a battery of intake questionnaires as part of standard clinical care within the PHP. Each week in the program, youths also completed a subset of the initial battery of questionnaires as follow-up. When participants consented to participate in the current study, these questionnaires, as well as clinical information from the electronic medical record, were retained and used for study purposes. No incentives were given for participation. Because of the complexity of collecting clinical measures on a busy unit with brief lengths of stay and moderate rates of informed consent, of the 283 youths that were admitted between August 5th, 2016, and December 31, 2018, data were only available for 158 patients or $55.8 \%$. Seventy youths were discharged before both the youth and the caregiver supplied consent to the study. Twelve youths had caregivers that did not speak English and therefore could not provide consent. Forty-three youths were not included from the present study analyses because they declined, or their caregivers declined to participate. One common reason for declining to participate in the study was privacy concerns $(n=15$, $34.8 \%$ ).

\section{Measures}

\section{Clinician-Rated Diagnoses}

Diagnostic information was collected from the youth's electronic medical record after discharge from the PHP. This was done to capture the team's diagnostic impressions after a course of treatment within the partial program, rather than the team's initial impressions at admission. Diagnoses, therefore, were informed by the team's initial clinical assessment, paired with information from individual and family sessions, collateral information, and direct observations of the youth during treatment within the PHP. Following data extrac- 
tion, diagnoses were coded into diagnostic categories following the classification system used in the Diagnostic and Statistical Manual of Mental Disorders - 5th Edition (American Psychiatric Association, 2013). When participants had more than one diagnosis listed on the discharge summary, all diagnoses were included in analyses examining frequencies.

\section{Screen for Child Anxiety Related Emotions Disorders, Child and Parent Versions (SCARED-C/P)}

The SCARED-C (Birmaher et al., 1997) is a 41-item self-report measure used to screen for signs of anxiety disorders in children. The SCARED-P is the corresponding parent-report version. Items are scored on a 4-point Likert-type scale from 0 (not true or hardly every true) to 3 (Very true or often true). The measure yields a composite total anxiety score as well as subscales for panic disorder, generalized anxiety, separation anxiety, social phobia, and school avoidance. Composite total anxiety scores greater than 25 suggest the presence of a youth anxiety disorder. The SCARED-C/P is a reliable and valid instrument to screen for childhood anxiety disorders in clinical settings, with good internal consistency $(\alpha=0.7-0.9)$, test-retest reliability $(p=0.6-0.9)$, and discriminant validity, both between anxiety and other psychiatric disorders and within anxiety disorders (Birmaher et al. 1997).

\section{Statistical Analysis}

\section{Analytic Plan}

Descriptive analyses were performed to describe the frequency of each anxiety and related disorder among the sample. From this point forward, "anxiety disorders" refers to social anxiety disorder, separation anxiety disorder, GAD, specific phobia, panic disorder, agoraphobia, and unspecified anxiety disorder, along with the following anxiety-related disorders: posttraumatic stress disorder (PTSD), adjustment disorder, obsessive-compulsive disorder (OCD) / body dysmorphic disorder (BDD), and trichotillomania (TTM). Thus, the frequency of anxiety disorders in this paper encompasses all the DSM-5 anxiety disorders grouped together with the anxiety-related disorders delineated above.

The frequency of anxiety disorders was calculated by identifying the percentage of participants in the sample with at least one anxiety disorder listed on their diagnostic profile. Frequency of depressive disorders was the percentage of participants in the sample with a diagnosis of major depressive disorder, persistent depressive disorder, or unspecified depressive disorder. Additionally, the frequency of comorbid anxiety disorders and depression was calculated by including any participant with at least one anxiety disorder and a depressive disorder. To parse the varied presentations of anxiety and depressive disorder status, frequencies were also calculated for the following groups: participants with at least one anxiety disorder, but no depressive disorder; participants with a depressive disorder, but no anxiety disorder; participants with neither an anxiety disorder nor depressive disorder.

Descriptive statistics were calculated to examine the mean and standard deviation for the SCARED-C and SCARED-P scores among the diagnostic groups. All analyses were conducted using IBM SPSS Statistics (Version 18.0). 


\section{Handling Missing Data}

When possible, analyses were based on the entire sample of 158 youths. No diagnostic data were missing from the electronic medical record. When available, the SCARED-C/P scores from the intake assessment were used. If available, SCARED-C scores from the week following the intake appointment were substituted for missing intake SCARED-C data. Following guidelines outlined by the developer of the SCARED, measures missing more than $20 \%$ ( $\geq 8$ items) were excluded from the analyses. Thus, SCARED-C means were generated from a final sample size of 145 youths and analyses involving the SCARED-P were computed using a sample of 135 caregivers. See Fig. 1 for a flow chart detailing missing data. Of the analyzed sample, missing data on items from the SCARED-C/P included in the study ranged from 0.41 to $2.46 \%$ (1-6 items). Mean imputation methods were explored to manage missing SCARED-C/P data but were not reported because the pattern of results with and without imputation did not differ substantially.

\section{Results}

\section{Primary Aim: Frequency of Clinician-Rated Anxiety Diagnoses}

Figure 2 illustrates the rates of anxiety and related disorders among adolescents in the sample. Among the 158 youths, GAD was the most diagnosed anxiety disorder $(30 \% ; n=47)$, followed by social anxiety disorder $(20 \% ; n=32)$, unspecified anxiety disorder $(20 \% ; n=$ $31)$, panic disorder $(6 \% ; n=10)$, separation anxiety disorder $(4 \% ; n=6)$, and agoraphobia $(1 \%, n=2)$. OCD was present in $8 \%$ of the sample $(n=13)$ and PTSD in $4 \%$ of the sample $(n=6)$. TTM $(1 \% ; n=2)$ and adjustment disorder $(2 \% ; n=3)$ were less common. In total, three-fourths $(75 \% ; n=118)$ of the adolescents in the PHP had at least one anxiety disorder diagnosis. Anxiety was equally as common as clinician-reported depressive disorders, which were represented in $72 \%(n=114)$ of the sample. Other diagnoses included attentiondeficit/hyperactivity disorder $(14 \% ; n=22)$, bipolar disorder $(7 \% ; n=11)$, substance use disorder $(4 \% ; n=7)$, learning disorder $(4 \% ; n=6)$, psychotic disorder $(3 \% ; n=4)$, disruptive behavior disorders $(3 \% ; n=4)$, Tourette syndrome $(1 \% ; n=2)$, autism spectrum disorder $(1 \% ; n=2)$, gender dysphoria $(1 \% ; n=2)$, eating disorder $(1 \% ; n=1)$, and somatic symptom disorder $(1 \% ; n=1)$.

Comorbidity was common (73\%) in this sample, with $48 \%(n=76)$ having two diagnoses, $15 \%(n=24)$ having three diagnoses and $9 \%(n=15)$ having four diagnoses. Figure 3 displays the percentage of participants with anxiety and depressive disorders as well as their combination. Over half $(54 \% ; n=85)$ of the participants had comorbid anxiety and depressive disorders, and approximately one-fifth of the sample had anxiety disorders without depressive disorders and vice versa $(21 \%$ and $18 \%$, respectively). Only $7 \%(n=11)$ of youths in the PHP had diagnoses that were neither anxiety nor depressive disorders. 


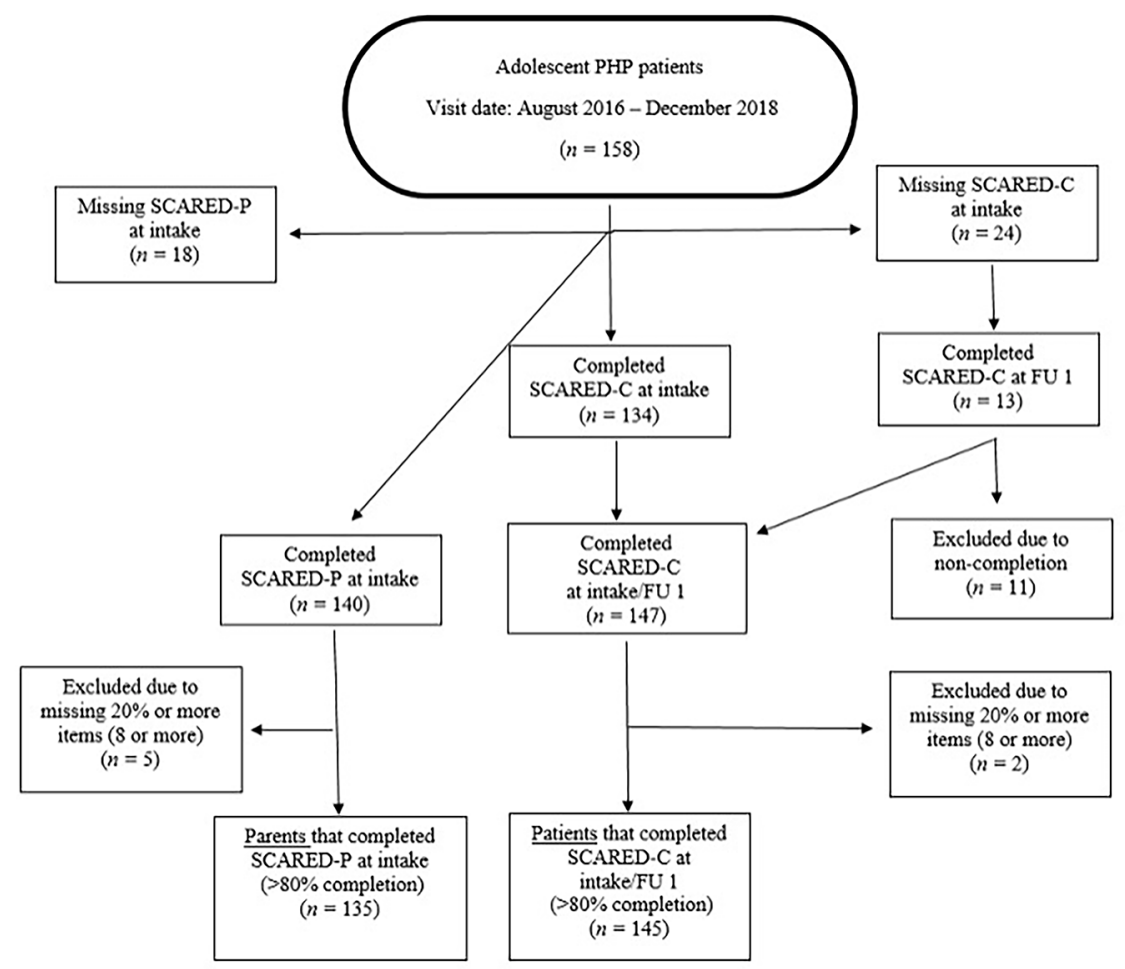

Fig. 1 Flow chart for missing data. Note: PHP = Partial Hospitalization Program, FU 1 = Follow up one assessment at 1 week

\section{Secondary Aim: Severity of Self- and Caregiver-Reported Anxiety Symptoms Among Youths with and without Diagnoses of Anxiety Disorders}

Figure 4 describes the severity of self- and parent-reported anxiety symptoms among those with and without diagnoses of anxiety disorders. On average, participants with anxiety disorders had SCARED-C scores that exceeded the clinical cutoff. Participants with depressive disorders also tended to have elevated SCARED-C scores above the clinical cutoff. When groups of youths with and without anxiety and depressive disorders were examined, it was found that youths with depressive disorders had elevated SCARED-C scores even when they did not meet criteria for a clinician-rated anxiety disorder in the electronic medical record. The only group with subthreshold anxiety symptoms on the SCARED-C were those participants with neither a depressive nor an anxiety disorder.

Caregiver ratings of the youth's anxiety symptoms on the SCARED-P were elevated above the clinical cut-off for youths with anxiety disorders. Youths with co-occurring anxiety and depressive disorders also exhibited elevated scores on the caregiver report measure. When youths presented with depressive disorders without an anxiety disorder, however, the SCARED-P scores fell below the clinical cutoff $(M=21.81 ; S D=16.96)$. 


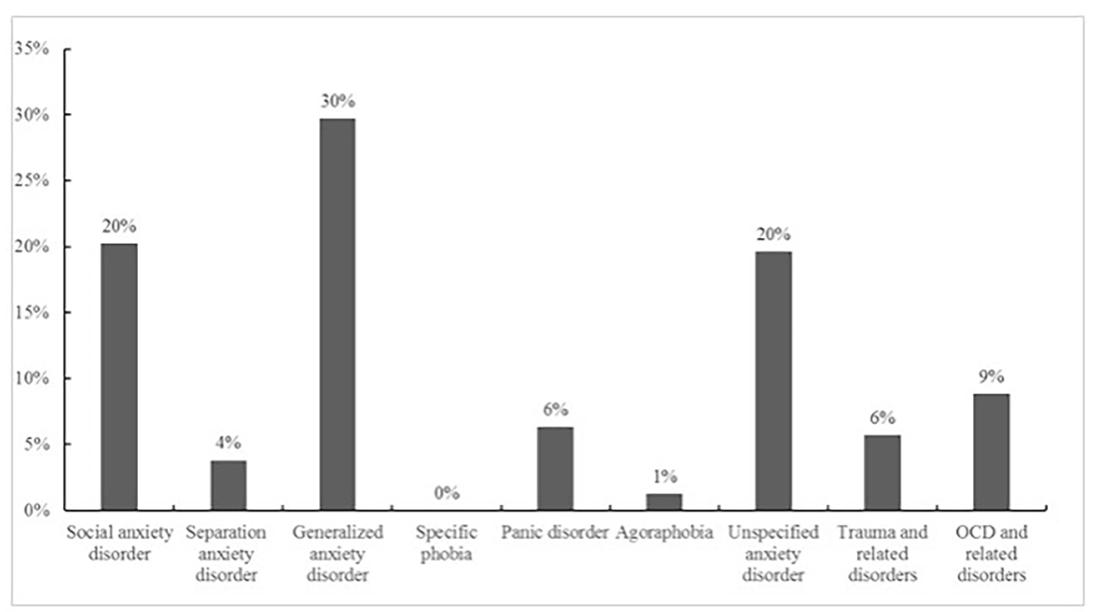

Fig. 2 Rates of youth anxiety disorders in the adolescent partial hospitalization program. Note: $\mathrm{N}=158$. Disorders are not mutually exclusive. Trauma and related disorders includes post-traumatic stress disorder, acute stress disorder and adjustment disorder. OCD and related disorders include OCD, BDD, and TTM

\section{Discussion}

The primary purpose of this study was to establish the frequency and severity of anxiety symptoms and anxiety disorders in an acute psychiatric care setting. These results indicate that anxiety symptoms are common in the partial hospitalization setting in which this study took place. The rate of anxiety and anxiety-related disorders (PTSD, OCD and TTM) was equivalent to the rate of depressive disorders in this population. Furthermore, patient- and parent-reported symptoms of anxiety were high, even for those patients who did not have a formal anxiety disorder diagnosis. Caregivers also endorsed anxiety symptoms in their youths with depression, but to a lesser degree. These findings suggest that anxiety is a common component of the psychiatric presentation in adolescent PHP patients that may require further attention and intervention. This section will discuss the implications of these findings as it relates to risk, the growing frequency of anxiety globally during the COVID-19 pandemic, the comorbidity of anxiety and depression, transdiagnostic considerations for assessment and treatment, study limitations, and clinical significance.

\section{Anxiety Disorders and Risk Presentations in Acute Care Settings}

Findings from this study suggest that anxiety is neither rare nor insignificant in partial hospitalization levels of care. Given the association between anxiety and common reasons for admission to a PHP, including suicidal ideation (Brown et al., 2018), suicide attempts (Hawton et al., 2013), substance abuse (Vorspan et al., 2015), and/or significant functional impairments (e.g., inability to engage in school or work; Gonzálvez et al., 2018), it is imperative to assess the presence, function, and role of anxiety in a patient's overall presentation upon admission to a PHP setting. While some hold that anxiety disorders do not contribute 


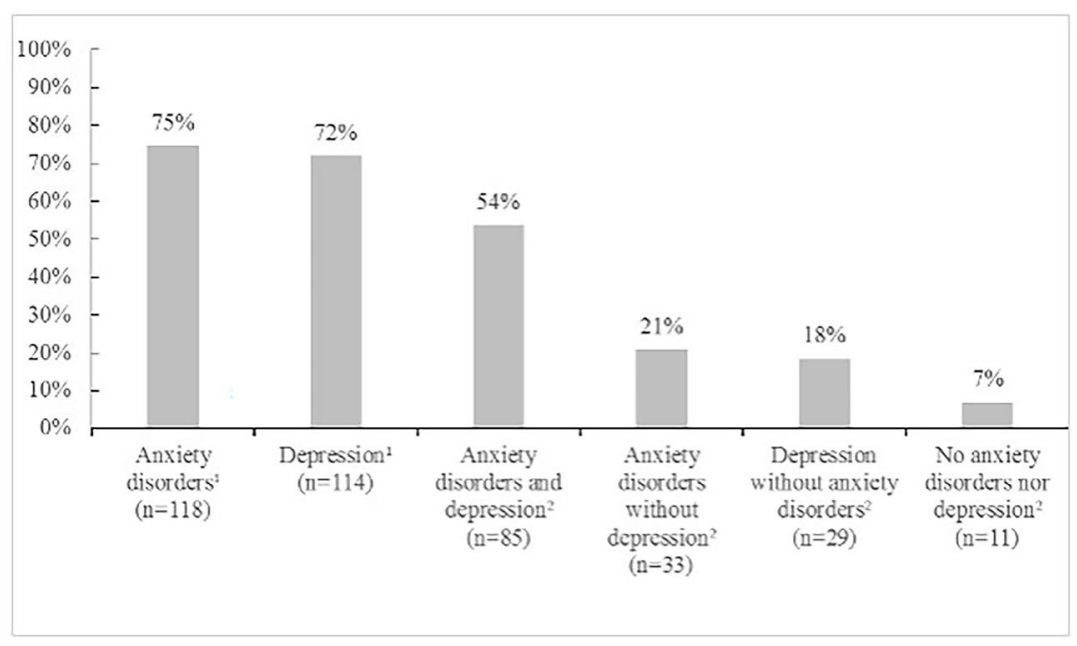

Fig. 3 Frequency rates of anxiety and depressive disorders among adolescent partial hospitalization patients. Note: $\mathrm{N}=158{ }^{1}$ Diagnostic groups are not mutually exclusive. ${ }^{2}$ Diagnostic groups are mutually exclusive

a significant risk for suicidal ideation, attempts, or death by suicide (Abreu et al., 2018), preliminary findings from another study from the PHP sampled here (Chiu et al., in prep) exploring consumer-nominated chief concerns among youths receiving acute care suggest that endorsement of suicidality as a top problem was related to higher severity of childreported anxiety symptoms. These findings add support to the notion that youth anxiety may be associated with increased reports of suicidality. This is consistent with a growing literature demonstrating considerable risk associations between anxiety disorders and suicide (de la Vega et al., 2018). The presence of both clinical and subclinical anxiety symptoms, anxiety sensitivity, and anxiety-driven behaviors have all been associated with suicidal ideation and risk (Anderberg et al., 2016; Stanley et al., 2018). Specific anxiety disorders (GAD and social anxiety disorder) have been found to be associated with higher risk for suicide (Bolton et al., 2008; Buckner et al., 2017; Foley et al., 2006), and anxiety is also a known risk factor for suicide in individuals with depression (Hawton et al., 2013).

\section{Severe Youth Anxiety and Depression Due to COVID-19}

This literature is made more concerning when seen in the context of rising anxiety, among other mental health concerns, worldwide. Since the outbreak of the COVID-19 pandemic, alarming psychiatric trends have emerged. Compared to U.S. adults in 2019, Americans were more than three times as likely to screen positively for depressive and/or anxiety disorders in April and May 2020 (Twenge \& Joiner, 2020). Furthermore, researchers at Harvard Medical School found that there have been distressing increases in anxiety, depression, and suicidal thoughts since the onset of the pandemic across multiple age groups, with youth being most vulnerable to these increases (Perlis et al., 2021). Students from lower income backgrounds have been particularly impacted by an increase in psychological dis- 


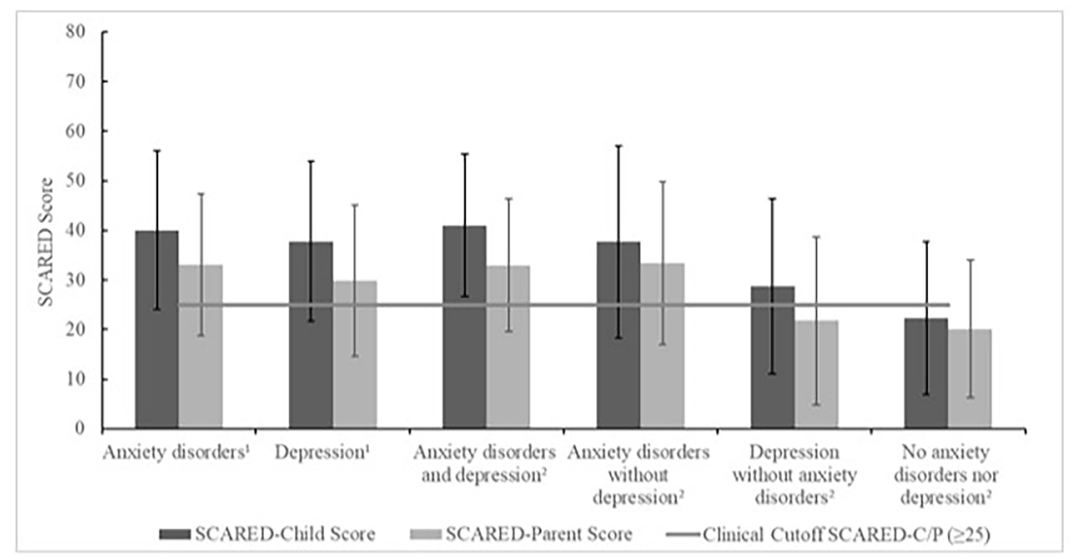

Fig. 4 Self and parent reported anxiety symptoms on the SCARED by diagnostic group. Note: SCARED$\mathrm{C} / \mathrm{P}$ : The screen for child anxiety and related disorders child version/parent versionThe $\mathrm{N}$ for each category varies from what was reported in Fig. 1 due to missing 1 to $19 \%$ SCARED-C/P. ${ }^{1}$ Diagnostic groups are not mutually exclusive. ${ }^{2}$ Diagnostic groups are mutually exclusive

tress since the beginning of the pandemic (Rudenstine et al., 2021). Similar trends are being observed in varied populations internationally, including China (Chen, et al., 2021), Ireland (Hyland et al., 2020), Italy (Simonetti et al., 2021), Spain (Günther-Bel et al., 2020), Turkey (Korukcu et al., 2021), and Canada, the United Kingdom, and Pakistan (Shah et al., 2021). In fact, a meta-analysis examining the global prevalence of youth anxiety and depression since the beginning of the pandemic estimates that anxiety and depression in children and adolescents have doubled and are continuing to rise worldwide (Racine et al., 2021). While admissions to higher levels of psychiatric care and suicide rates initially decreased during the pandemic (Pirkis et al., 2021; Tandon 2021; Ugueto \& Zeni, 2021), previous epidemics have demonstrated that suicide rates and hospitalizations may initially appear to decrease but may increase thereafter (Leaune et al., 2020; Zortea et al., 2021). Recent data indicates that mental health related emergency department visits among adolescents have increased precipitously relative to rates observed before the COVID-19 outbreak (Leeb et al. 2020, Yard et al., 2021), signaling the need for mental health professionals to be on high alert at this stage of the pandemic. With the global prevalence of anxiety and depression on the rise during this vulnerable time, particular attention must be paid to the assessment and treatment of these symptoms when they require higher levels of care. While the current study was completed before the pandemic, this overall thesis appears even more pressing considering this youth mental health crisis.

\section{Youth and Parent Reports of Anxiety and Depression}

Assessment and intervention broadly targeting the common comorbidity of anxiety and depression might better serve acute care settings. As highlighted in the current sample, anxiety and depression in youth have a complex and varied relationship. The finding that 
patients with no clinician-rated anxiety disorder diagnosis still self-endorse severe anxiety symptoms on the SCARED further confirms this complexity and the overlap between these clinical presentations. Caregivers also endorsed anxiety symptoms in their youth with depression, albeit to a lesser degree than their children. Studies on the discriminant validity of the SCARED suggest that the SCARED-C total score can differentiate youths with anxiety disorders from those with depressive disorders alone (Birmaher et al., 1997; Monga et al., 2000), such that youths with a depressive disorder and no diagnosed anxiety disorder tend to score lower on the SCARED-C. This was not the case in the present sample. It is possible that youths with a clinician-rated depressive disorder and no anxiety disorder were still experiencing elevated anxiety symptoms worthy of some intervention but perhaps not reaching threshold for a separate diagnosis. Parent report on the SCARED-P for youths with depression only did not exceed the clinical cut off however, suggesting that anxiety symptoms may have been more internally experienced by these youths and/or that depression was the predominant overt issue impairing functioning. There was some consistent discrepancy between youth and parent reports on the SCARED, which is common in self-report measures, including on the SCARED (Sequeira et al., 2019; van Doorn et al., 2018), though it did not fall outside the margin of error for the present sample. The frequency of youth and parent reported anxiety symptoms across all diagnostic categories underscores the utility of providing anxiety focused interventions and varied assessments for youths and parents in this setting.

There are multiple possible explanations for the common comorbidity of youth anxiety and depression in this population. Chronic anxiety and associated functional impairments can result in demoralization with symptoms of depression, rather than an independent mood disorder (Cummings et al., 2014). Additionally, there are global deficits commonly associated with both anxiety and depression, including difficulty regulating affect, tolerating distress, and controlling behavior (Trosper et al., 2009). Future studies might use longitudinal methods to further analyze the pathways explaining the comorbidity of anxiety and depression. This type of analysis was beyond the scope of the present dataset, as was teasing apart anxiety diagnoses as a primary versus secondary problem, due to the nature of data collection via general chart review. However, regardless of the explanatory pathway for this multifaceted relationship, the high rates of anxiety in the present sample call attention to anxiety as a clinical matter that requires specific assessment and intervention.

\section{Improving Anxiety Assessment and Intervention}

Given the high frequency and severity of anxiety in the present study, clinicians working in high acuity settings such as adolescent PHPs should be routinely assessing for anxiety disorders in addition to careful assessment of the patient's reason for referral and chief complaints. The risk factors associated with the presence of anxiety should be part of the requisite training for providers in PHP settings, and the appropriate targeted evidence-based interventions (e.g., CBT, DBT, and psychopharmacology) should be included in the milieu. PHP clinicians should keep in mind how anxiety symptoms may impact a patient's participation in PHP program activities. Patients and families should be educated about the contributing role of anxiety to the overall presenting problem and clinical picture. Clinicians, patients, and parents should all be advised on the appropriate after care plans to effectively 
address anxiety; when anxiety is identified at this level of care, after care treatment teams are better able to focus future treatment.

The common deficits associated with both anxiety and depression, including difficulty regulating affect, tolerating distress, and controlling behavior, could be treated with evidence based, transdiagnostic interventions (e.g., Chu et al., 2016; Ehrenreich-May et al., 2017). Moreover, directly targeting anxiety using CBT for anxiety disorders has been shown to decrease the rate of reported suicidal ideation in clinical settings (Brown et al., 2018), and DBT has a growing evidence base for decreasing suicidal ideation and behaviors in adolescents (for a systematic review, see Kothgassner et al., 2021).

\section{Limitations}

These findings must be interpreted within the context of study limitations. Collecting clinical data in a fast-moving partial hospitalization setting with limited research funds impacted data collection, contributing to missing data. There were missing sociodemographic data due to $7.0-13.9 \%$ of the participants failing to provide responses on some or all the demographic background questionnaire, as well as lost data due to patients' or caregivers' opting not to participate in research following informed consent. Data collection methods and measures were selected in large part with feasibility in mind, to not overburden patients or practitioners within the PHP setting. However, it is possible that data from youths who were not approached for consent, did not complete questionnaires, or who declined consent may be notably different from the data in this study sample. As a result, any implications from the current study's findings must be considered with this in mind.

A further limitation to consider is the generalizability of these findings, given that data for the present study were collected from a single PHP setting. Nearly two thirds (59.4\%) of the participants in this sample were referred by regional inpatient units or emergency rooms, suggesting that the majority of participants in this sample received acute psychiatric care services prior to admission to the PHP. These statistics imply that youths who are admitted to this PHP may be similar to the pool of youths who receive other regional acute care services, and thus, findings may be relevant to the broader population of youths who are seen across acute psychiatric care settings. In order to replicate and improve the generalizability of the current findings, similar projects must be undertaken on a larger scale in PHPs and in acute care settings across the country.

The results of this study highlight the importance of evidence-based assessment. This study found that one-fifth of participants had a clinician-reported unspecified anxiety disorder. It is difficult to conclude whether the PHP team deferred to this vague diagnosis because patients did not meet all the criteria to warrant a more specific anxiety diagnosis or whether the team deemed that further assessment was required. Nevertheless, a finer grained analysis of specific anxiety symptoms is potentially indicated. Structured intake interview templates or published semi-structured interviews (e.g., the Anxiety Disorder Interview Schedule; ADIS-IV C/P; Silverman \& Albano, 2004) provide the requisite questions to determine the specific anxiety disorders present, the severity of the anxiety disorder(s), the affectdriven behaviors contributing to functional impairments, and the interpersonal interactions or behavioral consequences that may reinforce and/or maintain the symptom presentation. 
Despite the resource burden, future studies should aim to integrate a structured diagnostic clinical interview such as the ADIS to augment current knowledge about diagnostic rates.

\section{Clinical Significance}

In sum, these data suggest that anxiety is common in an adolescent PHP setting, both in discrete diagnostic categories and in the symptom profiles of patients with anxiety and/or depressive disorder diagnoses. In a landscape where there are alarming increases in anxiety symptoms among the nation's youth, carefully assessing and addressing anxiety in PHPs and acute care settings is important. Considering the role of anxiety and being prepared to address it can improve understanding of the emergent reasons for admission, resolve underlying pathology leading to risk behaviors and impaired functioning, and finally, prevent readmission and relapse. These findings support investing resources in evidence-based assessment and treatment of anxiety in PHPs and other acute care settings.

Acknowledgements The authors would like to acknowledge Dr. David Shaffer for his wisdom and forward thinking about the impact of anxiety disorders on the development of high-risk behaviors such as self-harm, suicidal behaviors, and other profound impairments in functioning. This paper serves as a tribute to his prolific contributions to the field of child psychiatry broadly and the NYP Youth Anxiety Center specifically, without which this work would not have be possible.

This work is supported by the New York Presbyterian Youth Anxiety Center. It could not have been completed without the efforts of our research and clinical team, with special acknowledgement to Kerian Beckford, B.A., Debra Faecher, LCSW, Shannon Gasparro, B.A., Justin Mohatt, M.D., Jaleesa Payne, M.B.A., Jenna Rosenberg Wolfson, L.C.S.W., Jessica Simberlund, M.D., Heather Stanish, N.P.P. and Khadijah Watkins, M.D., as well as former and current postdoctoral fellows, Corinne Catarozoli, Ph.D., Avital Falk, Ph.D., Stephanie Rohrig, Ph.D., Laura Skriner, Ph.D., Paul Sullivan, Ph.D., Andrea Temkin, Psy.D. and Elaina Zendegui, Psy.D. We would also like to acknowledge the patients and families whose participation made this work possible.

Author Contributions All authors contributed to the study conception and design. Material preparation and data collection were performed by Renae Beaumont, PhD, Jennifer Schild, BS and Payal Desai, MPH. Data analysis was performed by Payal Desai, MPH. The first draft of the manuscript was written by Michelle Pelcovitz, $\mathrm{PhD}$, Shannon Bennett, $\mathrm{PhD}$, and Angela Chiu, $\mathrm{PhD}$ and all authors commented on previous versions of the manuscript. All authors (or their representatives) read and approved the final manuscript.

Funding This work was supported by the New York Presbyterian Youth Anxiety Center.

Availability of Data and Material The data are not from an open data source. Our Institutional Review Board (IRB) does not permit sharing our data.

Code Availability Not applicable.

Conflicts of Financial Interests Shannon Bennett, Ph.D. receives royalties from Wolters Kluwer. John Walkup, M.D. receives royalties from Wolters Kluwer, Guilford Press, and Oxford University Press, serves on the Scientific Advisory Board of the Anxiety and Depression Association of America (ADAA), the Trichotillomania Learning Center, and the Tourette Association of America. Dr. Walkup also has received honoraria from the American Association of Pediatrics (AAP), the American Psychiatric Association (APA), and the American Association of Child and Adolescent Psychiatry (AACAP). The remaining authors have no conflicts to disclose. 


\section{Conflicts of Non-Financial Interests None.}

Ethics Approval This research has been reviewed and approved by the Weill Cornell Medicine IRB.

Consent to Participate Informed consent was obtained from all participants over the age of 18. Informed assent was obtained from all participants under the age of 18, and informed consent was obtained from their parent or guardian.

\section{References}

Abreu, L. N., Oquendo, M. A., Galfavy, H., Burke, A., Grunebaum, M. F., Sher, L. ... Lafer, B. (2018). Are comorbid anxiety disorders a risk factor for suicide attempts in patients with mood disorders? A twoyear prospective study. European Psychiatry, 47, 19-24

American Psychiatric Association. (2013). Diagnostic and statistical manual of mental disorders (DSM-5®). American Psychiatric Pub

Anderberg, J., Bogren, M., Mattisson, C., \& Brådvik, L. (2016). Long-term suicide risk in anxiety-the Lundby study 1947-2011. Archives of Suicide Research, 20(3), 463-475

Bandelow, B., \& Michaelis, S. (2015). Epidemiology of anxiety disorders in the 21 st century. Dialogues in Clinical Neuroscience, 17(3), 327

Baxter, A. J., Vos, T., Scott, K. M., Ferrari, A. J., \& Whiteford, H. A. (2014). The global burden of anxiety disorders in 2010. Psychological Medicine, 44(11), 2363

Birmaher, B., Khetarpal, S., Brent, D., Cully, M., Balach, L., Kaufman, J., \& Neer, S. M. (1997). The screen for child anxiety related emotional disorders (SCARED): Scale construction and psychometric characteristics. Journal of the American Academy of Child \& Adolescent Psychiatry, 36(4), 545-553

Bolton, J. M., Cox, B. J., Afifi, T. O., Enns, M. W., Bienvenu, O. J., \& Sareen, J. (2008). Anxiety disorders and risk for suicide attempts: findings from the Baltimore Epidemiologic Catchment area follow-up study. Depression and Anxiety, 25(6), 477-481

Brown, L. A., Gallagher, T., Petersen, J., Benhamou, K., Foa, E. B., \& Asnaani, A. (2018). Does CBT for anxiety-related disorders alter suicidal ideation? Findings from a naturalistic sample. Journal of Anxiety Disorders, 59, 10-16

Buckner, J. D., Lemke, A. W., Jeffries, E. R., \& Shah, S. M. (2017). Social anxiety and suicidal ideation: Test of the utility of the interpersonal-psychological theory of suicide. Journal of Anxiety Disorders, $45,60-63$

Chen, X., Qi, H., Liu, R., Feng, Y., Li, W., Xiang, M. ... Xiang, Y. T. (2021). Depression, anxiety and associated factors among Chinese adolescents during the COVID-19 outbreak: a comparison of two crosssectional studies. Translational Psychiatry, 11(1), 1-8

Chiu, A. W., Desai, P., Skriner, L., Catarozoli, C., Sullivan, P., \& Bennett, S. (in preparation). Youth top problems: Describing consumer-nominated treatment needs in an acute psychiatric sample

Chu, B. C., Crocco, S. T., Esseling, P., Areizaga, M. J., Lindner, A. M., \& Skriner, L. C. (2016). Transdiagnostic group behavioral activation and exposure therapy for youth anxiety and depression: Initial randomized controlled trial. Behaviour Research and Therapy, 76, 65-75

Courtney, D., Watson, P., Battaglia, M., Mulsant, B. H., \& Szatmari, P. (2020). COVID-19 impacts on child and youth anxiety and depression: challenges and opportunities. The Canadian Journal of Psychiatry, 65(10), 688-691

Craske, M., Stein, M., Eley, T., Milad, M., Holmes, A., Rapee, R., \& Wittchen, H. (2017). Anxiety disorders. Nature Reviews. Disease Primers, 3(1), 17024

Cummings, C. M., Caporino, N. E., \& Kendall, P. C. (2014). Comorbidity of anxiety and depression in children and adolescents: 20 years after. Psychological Bulletin, 140(3), 816

De La Vega, D., Giner, L., \& Courtet, P. (2018). Suicidality in subjects with anxiety or obsessive-compulsive and related disorders: recent advances. Current Psychiatry Reports, 20(4), 1-10

Del Conte, G., Lenz, A. S., \& Hollenbaugh, K. M. (2016). A pilot evaluation of dialectical behavior therapy for adolescents within a partial hospitalization treatment milieu. Journal of Child and Adolescent Counseling, 2(1), 16-32

Ehrenreich-May, J., Kennedy, S. M., Sherman, J. A., Bilek, E. L., Buzzella, B. A., Bennett, S. M., \& Barlow, D. H. (2017). Unified protocols for transdiagnostic treatment of emotional disorders in children and adolescents: Therapist guide. Oxford University Press 
Foley, D. L., Goldston, D. B., Costello, E. J., \& Angold, A. (2006). Proximal psychiatric risk factors for suicidality in youth: the Great Smoky Mountains Study. Archives of General Psychiatry, 63(9), 1017-1024

Gonzálvez, C., Kearney, C. A., Jiménez-Ayala, C. E., Sanmartín, R., Vicent, M., Inglés, C. J., \& GarcíaFernández, J. M. (2018). Functional profiles of school refusal behavior and their relationship with depression, anxiety, and stress. Psychiatry Research, 269, 140-144

Gould, M. S., Greenberg, T. E. D., Velting, D. M., \& Shaffer, D. (2003). Youth suicide risk and preventive interventions: a review of the past 10 years. Journal of the American Academy of Child \& Adolescent Psychiatry, 42(4), 386-405

Günther-Bel, C., Vilaregut, A., Carratala, E., Torras-Garat, S., \& Pérez-Testor, C. (2020). A mixed-method study of individual, couple, and parental functioning during the state-regulated COVID-19 lockdown in Spain. Family Process, 59(3), 1060-1079

Hallfors, D. D., Waller, M. W., Ford, C. A., Halpern, C. T., Brodish, P. H., \& Iritani, B. (2004). Adolescent depression and suicide risk: association with sex and drug behavior. American Journal of Preventive Medicine, 27(3), 224-231

Hawton, K., i Comabella, C. C., Haw, C., \& Saunders, K. (2013). Risk factors for suicide in individuals with depression: a systematic review. Journal of Affective Disorders, 147(1-3), 17-28

Hyland, P., Shevlin, M., McBride, O., Murphy, J., Karatzias, T., Bentall, R. P. ... Vallières, F. (2020). Anxiety and depression in the Republic of Ireland during the COVID-19 pandemic. Acta Psychiatrica Scandinavica, 142(3), 249-256

Kessler, R. C., Petukhova, M., Sampson, N. A., Zaslavsky, A. M., \& Wittchen, H. U. (2012). Twelve-month and lifetime prevalence and lifetime morbid risk of anxiety and mood disorders in the United States. International Journal of Methods in Psychiatric Research, 21(3), 169-184

Korukcu, O., Ozkaya, M., Boran, F., O., \& Boran, M. (2021). The effect of the COVID-19 pandemic on community mental health: A psychometric and prevalence study in Turkey. Health \& Social Care in the Community, 29, e204-e213

Kothgassner, O., Goreis, A., Robinson, K., Huscsava, M., Schmahl, C., \& Plener, P. (2021). Efficacy of dialectical behavior therapy for adolescent self-harm and suicidal ideation: A systematic review and meta-analysis. Psychological Medicine, 51(7), 1057-1067

Leaune, E., Samuel, M., Oh, H., Poulet, E., \& Brunelin, J. (2020). Suicidal behaviors and ideation during emerging viral disease outbreaks before the COVID-19 pandemic: a systematic rapid review.Preventive Medicine, 106264

Leeb, R. T., Bitsko, R. H., Radhakrishnan, L., Martinez, P., Njai, R., \& Holland, K. M. (2020). Mental healthrelated emergency department visits among children aged $<18$ years during the COVID-19 pandemicUnited States, January 1-October 17, 2020. Morbidity Mortality Weekly Report 2020, 69(45), 1675-80

Leffler, J. M., Junghans-Rutelonis, A. N., \& McTate, E. A. (2020). Feasibility, acceptability, and considerations for sustainability of implementing an integrated family-based partial hospitalization program for children and adolescents with mood disorders. Evidence-Based Practice in Child and Adolescent Mental Health, 5(4), 383-397

Martino, M., Schneider, B. N., Park, M., Podell, J. L., \& Peris, T. S. (2020). Evidence-based practice in a pediatric partial hospitalization setting. Evidence-Based Practice in Child and Adolescent Mental Health, 5(1), 28-41

Mee-Lee, D., \& Shulman, G. D. (2003). The ASAM placement criteria and matching patients to treatment. Principles of Addiction Medicine. Chevy Chase, MD: American Society of Addiction Medicine, Inc

Merikangas, K. R., He, J. P., Burstein, M., Swanson, S. A., Avenevoli, S., Cui, L. ... Swendsen, J. (2010). Lifetime prevalence of mental disorders in US adolescents: results from the National Comorbidity Survey Replication-Adolescent Supplement (NCS-A). Journal of the American Academy of Child \& Adolescent Psychiatry, 49(10), 980-989

Monga, S., Birmaher, B., Chiappetta, L., Brent, D., Kaufman, J., Bridge, J., \& Cully, M. (2000). Screen for child anxiety-related emotional disorders (SCARED): Convergent and divergent validity. Depression and Anxiety, 12(2), 85-91

Naragon-Gainey, K., Gallagher, M. W., \& Brown, T. A. (2014). A longitudinal examination of psychosocial impairment across the anxiety disorders. Psychological Medicine, 44(8), 1691-1700

O’Sullivan, K., Clark, S., McGrane, A., Rock, N., Burke, L., Boyle, N. .. Marshall, K. (2021). A qualitative study of child and adolescent mental health during the COVID-19 pandemic in Ireland. International Journal of Environmental Research and Public Health, 18(3), 1062

Perlis, R. H., Green, J., Quintana, A., Gitomer, A., Chwe, H., Simonson, M. ... Druckman, J. (2021, February 10). The COVID States Project \#23: Depression among young adults. https://doi.org/10.31219/osf. io/3s $8 v 4$

Pirkis, J., John, A., Shin, S., DelPozo-Banos, M., Arya, V., Analuisa-Aguilar, P. ... Bertolote, J. M. (2021). Suicide trends in the early months of the COVID-19 pandemic: an interrupted time-series analysis of preliminary data from 21 countries. The Lancet Psychiatry, 8(7), 579-588 
Polanczyk, G. V., Salum, G. A., Sugaya, L. S., Caye, A., \& Rohde, L. A. (2015). Annual research review: A meta-analysis of the worldwide prevalence of mental disorders in children and adolescents. Journal of Child Psychology and Psychiatry, 56(3), 345-365

Racine, N., McArthur, B. A., Cooke, J. E., Eirich, R., Zhu, J., \& Madigan, S. (2021). Global prevalence of depressive and anxiety symptoms in children and adolescents during COVID-19: A meta-analysis. JAMA Pediatrics

Robinson (2000). Outcomes of a Partial-Day Treatment Program for Referred Children. Child and Youth Care Forum, 29, 127-137

Robinson, K. E., Dow, R. T., \& Nicholas, P. M. (1999). Expanding a continuum of care: A report on a partial day treatment program. Child \& Youth Care Forum, 28(3), 221-228

Rudenstine, S., McNeal, K., Schulder, T., Ettman, C. K., Hernandez, M., Gvozdieva, K., \& Galea, S. (2021). Depression and anxiety during the covid-19 pandemic in an urban, low-income public university sample. Journal of Traumatic Stress, 34(1), 12-22

Sequeira, S. L., Silk, J. S., Woods, W. C., Kolko, D. J., \& Lindhiem, O. (2019). Psychometric properties of the SCARED in a nationally representative US sample of 5-12-year-olds. Journal of Clinical Child \& Adolescent Psychology

Shaffer, D., Gould, M. S., Fisher, P., Trautman, P., Moreau, D., Kleinman, M., \& Flory, M. (1996). Psychiatric diagnosis in child and adolescent suicide. Archives of General Psychiatry, 53(4), 339-348

Shah, S. M. A., Mohammad, D., Qureshi, M. F. H., Abbas, M. Z., \& Aleem, S. (2021). Prevalence, Psychological Responses and associated correlates of depression, anxiety and stress in a global population, during the coronavirus disease (COVID-19) pandemic. Community Mental Health Journal, 57(1), 101-110

Silverman, W. K., \& Albano, A. M. (2004). Anxiety Disorders Interview Schedule for DSM-IV (ADIS-IV): Child and Parent Interview Schedules. San Antonio, TX: Psychological Corporation

Simonetti, V., Durante, A., Ambrosca, R., Arcadi, P., Graziano, G., Pucciarelli, G. ... Cicolini, G. (2021). Anxiety, sleep disorders and self-efficacy among nurses during COVID-19 pandemic: A large cross-sectional study. Journal of Clinical Nursing, 30(9-10), 1360-1371

Stanley, I. H., Boffa, J. W., Rogers, M. L., Hom, M. A., Albanese, B. J., Chu, C. ... Joiner, T. E. (2018). Anxiety sensitivity and suicidal ideation/suicide risk: A meta-analysis. Journal of Consulting and Clinical Psychology, 86(11), 946

Substance Abuse and Mental Health Services Administration (2019). National Mental Health Services Survey (N-MHSS): 2018. Data on Mental Health Treatment Facilities. Rockville, MD: Substance Abuse and Mental Health Services Administration

Tandon, R. (2021). COVID-19 and suicide: Just the facts. Key learnings and guidance for action. Asian Journal of Psychiatry, 60 (2021), 102695

Thatte, S., Makinen, J. A., Nguyen, H. N., Hill, E. M., \& Flament, M. F. (2013). Partial hospitalization for youth with psychiatric disorders: treatment outcomes and 3-month follow-up. The Journal of Nervous and Mental Disease, 201(5), 429-434

Trosper, S. E., Buzzella, B. A., Bennett, S. M., \& Ehrenreich, J. T. (2009). Emotion regulation in youth with emotional disorders: implications for a unified treatment approach. Clinical Child and Family Psychology Review, 12(3), 234-254

Twenge, J. M., \& Joiner, T. E. (2020). US Census Bureau-assessed prevalence of anxiety and depressive symptoms in 2019 and during the 2020 COVID-19 pandemic. Depression and Anxiety, 37(10), 954-956

Ugueto, A. M., \& Zeni, C. P. (2021). Patterns of youth inpatient psychiatric admissions before and after the onset of the COVID-19 pandemic. Journal of the American Academy of Child and Adolescent Psychiatry, 60(7), 796-798

Van Doorn, M. M. E. M., Bodden, D., Jansen, M., Rapee, R. M., \& Granic, I. (2018). Linking mother-child discrepancies to behavioral observations of children's anxiety. Child \& Youth Care Forum, 47, 481-498

Vorspan, F., Mehtelli, W., Dupuy, G., Bloch, V., \& Lépine, J. P. (2015). Anxiety and substance use disorders: co-occurrence and clinical issues. Current Psychiatry Reports, 17(2), 4

Woodward, L. J., \& Fergusson, D. M. (2001). Life course outcomes of young people with anxiety disorders in adolescence. Journal of the American Academy of Child \& Adolescent Psychiatry, 40(9), 1086-1093

Yard, E., Radhakrishnan, L., Ballesteros, M. F., Sheppard, M., Gates, A., Stein, Z. ... Ehlman, D. C. (2021). Emergency department visits for suspected suicide attempts among persons aged 12-25 years before and during the COVID-19 pandemic-United States, January 2019-May 2021. Morbidity and Mortality Weekly Report, 70(24), 888

Zander, E., Wyder, L., Grosse Holtforth, M., Schnyder, U., Hepp, U., \& Stulz, N. (2018). Validity of routine clinical diagnoses in acute psychiatric inpatients. Psychiatry Research, 259, 482-487

Zortea, T., Brenna, C., Joyce, M., McClelland, H., Tippett, M., Tran, M. ... Platt, S. (2021). The impact of infectious disease-related public health emergencies on suicide, suicidal behavior, and suicidal thoughts. Crisis: The Journal of Crisis Intervention and Suicide Prevention, 42(6), 474-487 
Publisher's Note Springer Nature remains neutral with regard to jurisdictional claims in published maps and institutional affiliations.

\section{Authors and Affiliations}

Michelle Pelcovitz ${ }^{1}$.Shannon Bennett ${ }^{1} \cdot$ Payal Desai $^{2} \cdot$ Jennifer Schild ${ }^{1}$. Renae Beaumont ${ }^{1}$. John Walkup ${ }^{3}$. David Shaffer ${ }^{4}$. Angela Chiu ${ }^{1}$

Michelle Pelcovitz

mip2051@med.cornell.edu

1 Department of Psychiatry, Weill Cornell Medicine, New York-Presbyterian Hospital, New York, United States

2 New York State Psychiatric Institute, Columbia University, New York, United States

3 Department of Psychiatry, Lurie Children's Hospital, Chicago, United States

4 Department of Psychiatry, Columbia University Medical Center, New York, United States 